\title{
Color Iris Authentication using Color Models
}

\author{
Abbhilasha S. Narote \\ S.K.N. College of Engineering, \\ Pune, India
}

\author{
Laxman M. Waghmare \\ S.G.G.S. Institute of \\ Engineering \& Technology \\ Nanded, India
}

\begin{abstract}
Iris authentication is a popular method where persons are accurately authenticated. During authentication phase the features are extracted which are unique. Iris authentication uses IR images for authentication. This proposed work uses color iris images for authentication. Experiments are performed using ten different color models. This paper is focused on performance evaluation of color models used for color iris authentication. This proposed method is more reliable which cope up with different noises of color iris images. The experiments reveals the best selection of color model used for iris authentication. The proposed method is validated on UBIRIS noisy iris database. The results demonstrate that the accuracy is $92.1 \%$, equal error rate of 0.072 and computational time is 0.039 seconds.
\end{abstract}

\section{Keywords}

Biometrics, iris recognition, authentication, feature extraction, matching.

\section{INTRODUCTION}

Iris recognition $[1,2,3]$ is widely accepted as one of the most stable and accurate reliable biometric technique. The iris patterns are very complex which contains many distinctive features such as radial furrows, concentric furrows, zigzag collarette, crypts, freckles, rings and corona $[3,4]$. In the last two decades, there is significant progress of use of iris recognition [4-7]. Compared with some other biometrics, iris has various advantageous factors such as greater speed, simplicity, and accuracy. Literature reveals that the irises of identical twins are different and also right and left irises of an individual are different. The properties of iris makes it as one of the most secure biometrics for identification and authentication. Iris recognition is widely accepted as one of the best biometrics recognition methods [3], in the world because of its stability, uniqueness and non-invasiveness. One of the key problem in iris recognition is feature extraction. This features should be highly distinctive between the iris images of different eyes while maintaining minimum distance between iris images of same eyes.

A generic iris recognition system [3] consists of image acquisition, iris segmentation, feature extraction, matching and recognition. For recognition /authentication of a person, the eye image is captured using infrared (IR) camera. While acquiring the eye image, eyes are illuminated using infrared light. The acquired image of the eye contains data, derived from the surrounding eye region along with iris. Therefore, initially the iris is localized in the segmentation module from the acquired image. The size of the individual iris goes on changing with respect to changes in illumination, also the size of iris each individual is different. In order to avoid inconsistency in the size of the iris, it is normalized. In the feature extraction module, features are extracted from the normalized iris. This features is encoded and then matched with the other template.
The limitations of this IR based iris authentication system is that the color information is lost and special IR cameras are required for acquiring iris images. This paper proposes a new method for iris extraction and also color histogram based approach is used for iris authentication. The color histogram information is directly adopted as features. Color histogram has simple mathematical analysis which reduces complex mathematical computations. The computational complexity of the feature extraction process is much lower than other filtering based approaches.

The remainder of the paper is organized as follows: Section 2 describes the preprocessing method. Section 3 describes the proposed method of iris feature extraction using histogram of color features. Section 4 describes the matching process of the proposed approach. Section 5 describes the result analysis and the conclusion is presented in Section 6 .

\section{IRIS PREPROCESSING}

Iris patterns are unique for a person which remains stable throughout the life [2,4]. The iris image of a person is acquired at different instant of time. During this instant the external environments are not same, i.e. the distance between the camera and eyes, tilting of head, eye movements and pupil dilation. All these factors need to be considered while feature extraction. Therefore prior, to feature extraction iris image need to be preprocessed.

The block diagram of proposed color iris authentication using color models is shown in Fig. 1. Iris segmentation plays a vital role in the performance of iris identification system. Several segmentation techniques have been proposed in the literature. The most two popular techniques used for iris segmentation are integro-differential operator [2] and the Hough transform [4] respectively. The first stage of iris segmentation is to find out pupil boundary and then the limbus boundary.
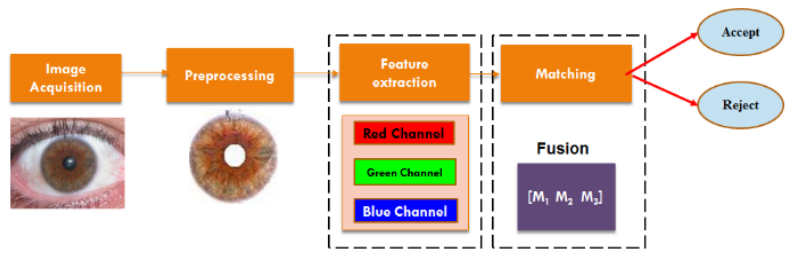

Fig 1 Block diagram of color iris authentication system

The UBIRIS [8], iris database consist of color images. The color eye image consist of RGB colors which is used for iris segmentation. The red channel (plane) of this color eye image is used for iris segmentation, which reduce the processing time required for conversion into gray level format. The pupil boundary and its radius are extracted using the histogram method [9]. The limbus boundary and its radius are detected using integro-differential operator [2]. The circular Hough transform is based on voting scheme which is tolerant to noise. To detect the limbus boundary of noisy iris images from UBIRIS database the circular Hough transform is 
selected for limbus boundary detection. In circular Hough transform the voting process is carried out in a parameter space. A circular Hough space is given in equation 1 as

$$
H\left(x_{c}, y_{c}, r\right)=\sum_{i=1}^{n} h\left(x_{i}, y_{i}, x_{c}, y_{c}, r\right)
$$

where $\mathrm{r}$ is radius, $\left(\mathrm{x}_{\mathrm{c}}, \mathrm{y}_{\mathrm{c}}\right)$ are circle center, $\left(\mathrm{x}_{\mathrm{i}}, \mathrm{y}_{\mathrm{i}}\right)$ is an edge point and $\left(\mathrm{x}_{\mathrm{o}}, \mathrm{y}_{\mathrm{o}}, \mathrm{r}\right)$ are location. $\mathrm{H}\left(\mathrm{x}_{\mathrm{c}}, \mathrm{y}_{\mathrm{c}}, \mathrm{r}\right)$ is an accumulator array. The maximum value of the accumulator is selected as the parameter for the largest circular boundary. This parameter gives the limbus center and radius value. Using pupil radius, limbus radius and center values, pupil boundary and limbus boundary are mapped on color iris as shown in Figure 2 .

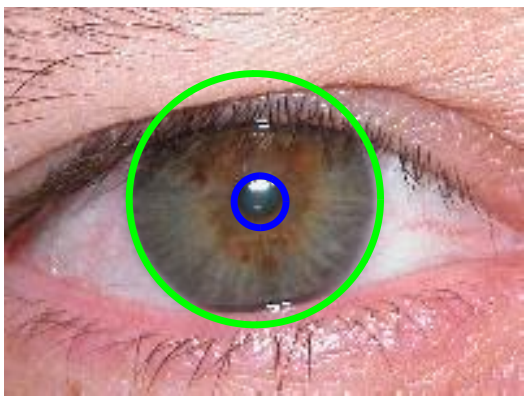

Fig 2. Segmeneted iris image

The segmented image consists of the original eye image on which pupil boundary and limbus boundary is mapped. The pixels inside the pupil boundary are not useful, which are considered as noise and similarly the portion outside limbus boundary are not useful which are also considered as noise. This noise from the segmented image is to be removed and only iris image is to be preserved. Here the pixels outside the limbus boundary and the pixels inside the pupil boundary are replaced with white pixels. The sample extracted iris image from segmented images are shown in Figure 3. The size of extracted iris image is different for every image.

There is pupil constriction and dilation due to illumination conditions, environmental, physiological factors, the distance between the camera and iris image, etc. This iris deformation will affect the overall performance during matching. In order to compensate this elastic deformation iris image is normalized.
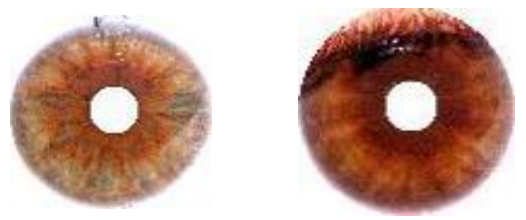

Fig 3. Extracted iris image

Normalization method transforms a localized iris texture from Cartesian to polar coordinates. The majority of the iris recognition algorithm uses rubber sheet model suggested by Daugman [2] for normalization. In this color iris authentication system normalization step is eliminated.

\section{IRIS FEATURE EXTRACTION USING HISTOGRAM OF COLOR MODELS}

The first iris recognition was proposed by Daugman [2] which uses Gabor filter for characterizing texture pattern,, where the phase information of the filtered image is encoded into binary pattern. Laplacian of Gaussian (LoG) filter was used by Wildes for feature extraction. In the literature, it has been observed that various filter bank techniques have been suggested by authors for iris feature extraction. An alternative to IR images based iris recognition is to use color information of iris for iris recognition. Krichen [10] used wavelet packets for iris identification using color iris images. Hugo [8] created a noisy iris database UBIRIS were gray scale images were used for experimentation. Boyce et. al. [11] used multispectral iris images for segmentation and recognition. Here RGB images are converted into Lab color space, for feature extraction 2D Gabor filters and radial feature vectors are used. Monaco [12] used color space analysis for iris recognition in which Gabor filters are used for feature extraction from each band. For color iris recognition and indexing Gabor filter and other filter bank techniques have been used. This filter banks provide excellent accuracy, but their design is complex and are computationally expensive.

The infrared iris image is a gray scale image were as color iris images consists of three channel data. The color image data is acquired from three sensors, i.e. red, green and blue. The RGB image is used for segmentation. Prior to feature extraction the segmented RGB image is to be converted to the desired different color space. A color space is a model used for representing color values in intensity. There are a number of color spaces available, from this a particular color space is selected depending upon the application. The RGB color model is the most popular model used for processing digital images. The RGB color space, describes colors by three components: red, green and blue. The disadvantage of RGB model is that gets effected due to intensity variations. The other color space models are transformed from the RGB model using linear and nonlinear transformation matrix such as YUV, YIQ, HSV, HIS, etc.

The HSV color model is a popular model for computer graphics. The hue of an image refers to a pure color. The hue ranges from $0-360^{\circ}$ where each value corresponds to one color. The saturation and value ranges from 0 to 1 where saturation is the intensity of the color and value is the measurement of brightness of color. Unlike other color models HSV is not device independent. HSV is popular due to its similarity with the way human perceive color.

Different color models have been used for iris recognition. Boyce et. al. [11] uses CIE Lab color space, Monaco [12] uses RGB, CIE lab, HSV, CMYK, YcbCrSun [13], Al-Quanieer [14], Tan [15] and Sibai [16] used RGB color models for preprocessing. RGB and nine different color models, i.e. YUV, YIQ, HSV, HIS, HSL, Lab, Luv, LCH and YCbCr are used for transforming segmented RGB iris image into different spaces. Based on experimentation five color models HSI, HSV, YIQ, Lab and RGB model are finally selected. Using this color model RGB extracted iris images are transformed into different color spaces. From this transformed image features are extracted. 


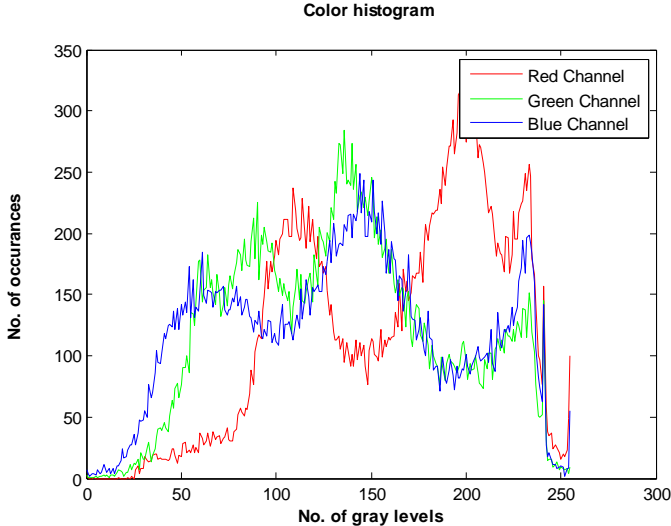

Fig 4. Color histogram of eye image

Color histogram is a standard method of extracting features from a color image. As color iris images are considered, the color histogram is used for feature extraction. The Color histogram of an image is rotation, translation, and scale invariant [17]. The color histogram is the probability distribution of color pixels in an RGB image defined as

$$
H_{(R, G, B)}=\operatorname{Probability}\left(N_{R, G, B}\right)
$$

This probability function is a color histogram $H_{R, G, B}$ where $\mathrm{H}_{\mathrm{R}}, \mathrm{H}_{\mathrm{G}}$ and $\mathrm{H}_{\mathrm{B}}$ represents the frequency of occurrences of red, green and blue channels respectively. For color images, the histogram is fragmented into three histograms of the three channels as $\mathrm{H}_{\mathrm{R}}, \mathrm{H}_{\mathrm{G}}$ and $\mathrm{H}_{\mathrm{B}}$ as shown in Figure 4.

\section{MATCHING}

Once the features are extracted, then these features are used for matching. As the features extracted from iris are independent from other channels, their respective levels are considered as feature vectors. There are various distances available to compare the similarity between two irises using this pdfs. For measuring the similarity distances Euclidean distance and Manhattan are used.

The Euclidean distance (ED) and Manhattan distance (MHD) is commonly used for similarity measurement due to its efficiency, effectiveness and less time for computation. The ED compares the similarity between two feature vectors of iris image by estimating the square root of the sum of the squared absolute differences as given in equation 3 . The value of $p$ is taken as 2 in Euclidean distance.

Euclidean Distance $E D_{(a, b)}=\sqrt{\sum_{i=1}^{d}\left(a_{i}-b_{i}\right)^{p}}$

Manhattan Distance (MHD) also called as city block distance measures the similarity between two iris image by taking the sum of the absolute values of the differences between the two feature vectors as shown in equation 4

ManhattanDistance $\operatorname{MHD}_{(a, b)}=\sum_{i=1}^{d}\left|a_{i}-b_{i}\right|$

Experiments are carried out using above distances. The computational complexity is different for different distance measures. This is computed based on number of multiplication, addition and subtraction. For a feature vector of size d. Euclidean distance requires d subtractions for ai bi, $d$ squares of the previous, $d-1$ further additions and one square root at the end. Similarly Manhattan distance requires $\mathrm{d}$ subtractions for ai $-\mathrm{bi}, \mathrm{d}-1$ addition. The time required for comparing two iris image is 0.000025 and 0.000021 secs using Manhattan and Euclidean distance.

\section{EXPERIMENTAL RESULTS}

To evaluate the effectiveness of the proposed method for color iris authentication, UBIRIS database is used. In this section set of experiments are conducted to evaluate the performance of the proposed method and summarize the results. The performance is evaluated in two stages: first based on segmentation, color transformation, feature extraction and pattern matching and second based on authentication mode. For authentication mode the performance is measured in terms of genuine accept rate (GAR), false match rate(FMR), false non match rate (FNMR) and equal error rate (EER). The experiments are conducted on a COREi5 system with 4 GB RAM and MATLAB 2014A environment.

The performance of the proposed algorithm is evaluated on the UBIRIS database [8]. The images from the database have been captured from 241 persons with 5 images for each user in first session resulting in total 1205 images with image resolution of $200 \times 150$. In a second session images are captured from 132 persons. The color images are acquired in visible light spectrum. The important characteristic of this database is that it incorporates several noise such as poor illumination, contrast, reflection, focus and occlusion in the iris images. Performance of segmentation method is tested on session one images in percentage using histogram and integrodifferential operator (IDO) and histogram and circular Hough transform(CHT) which is given in Table I. Using combination of histogram and circular Hough transform the segmentation accuracy achieved is $91.86 \%$

Table 1. Performance of different segmentation algorithm in percentage

\begin{tabular}{|c|c|}
\hline Segmentation Methodology & Segmentation\% \\
\hline Daugmans algorithm & 72.53 \\
\hline Masek Algorithm & 79.17 \\
\hline Histogram and IDO & 87.55 \\
\hline Histogram and CHT & 91.86 \\
\hline
\end{tabular}

Ten color models are used for experimentation, i.e. RGB, YUV, YIQ, HSV, HIS, HSL, Lab, Luv, LCH and YCbCr. From this ten color model, based on the performance, seven color models HSI, HSV, YIQ, Lab, Luv and RGB model are selected. Table 2 to Table 4 presents the EER for individual channel of HSV, HSI, YUV, YIQ, Lab and Luv color model.

Table 2. EER of HSV and HIS model for individual channel

\begin{tabular}{|c|c|c|}
\hline \multirow{2}{*}{ Color Model } & \multicolumn{2}{|c|}{ EER } \\
\cline { 2 - 3 } & MHD & ED \\
\hline HSI $_{\mathbf{1}}$ & 0.0218 & 0.2153 \\
\hline $\mathbf{H S V}_{\mathbf{1}}$ & 0.0218 & 0.2153 \\
\hline $\mathbf{H S I}_{\mathbf{2}}$ & 0.047 & 0.087 \\
\hline $\mathbf{H S V}_{\mathbf{2}}$ & 0.059 & 0.083 \\
\hline $\mathbf{H S I}_{\mathbf{3}}$ & 0.071 & 0.083 \\
\hline $\mathbf{H S V}_{\mathbf{3}}$ & 0.071 & 0.086 \\
\hline
\end{tabular}


Table 3. EER of YUV and YIQ model for individual channel

\begin{tabular}{|c|c|c|}
\hline \multirow{2}{*}{ Color Model } & \multicolumn{2}{|c|}{ EER } \\
\cline { 2 - 3 } & MHD & ED \\
\hline YUV $_{\mathbf{1}}$ & 0.0218 & 0.222 \\
\hline YIQ $_{\mathbf{1}}$ & 0.083 & 0.075 \\
\hline YUV $_{\mathbf{2}}$ & 0.253 & 0.222 \\
\hline YIQ $_{\mathbf{2}}$ & 0.051 & 0.052 \\
\hline $\mathbf{Y U V}_{\mathbf{3}}$ & 0.051 & 0.052 \\
\hline $\mathbf{Y I Q}_{\mathbf{3}}$ & 0.102 & 0.088 \\
\hline
\end{tabular}

Table 4. EER of Lab and Luv model for individual channel

\begin{tabular}{|c|c|c|}
\hline \multirow{2}{*}{ Color Model } & \multicolumn{2}{|c|}{ EER } \\
\cline { 2 - 3 } & MHD & ED \\
\hline Lab $_{\mathbf{1}}$ & 0.0218 & 0.232 \\
\hline Luv $_{\mathbf{1}}$ & 0.232 & 0.232 \\
\hline Lab $_{\mathbf{2}}$ & 0.222 & 0.210 \\
\hline Luv $_{\mathbf{2}}$ & 0.225 & 0.213 \\
\hline Lab $_{\mathbf{3}}$ & 0.227 & 0.217 \\
\hline Luv $_{\mathbf{3}}$ & 0.224 & 0.211 \\
\hline
\end{tabular}

In order to improve the performance of color models, the data is fused at score level. This score level fusion improves the EER. Figure 5 and 6 shows the DET curves for best five color models. Based on these DET curves the EER is estimated.

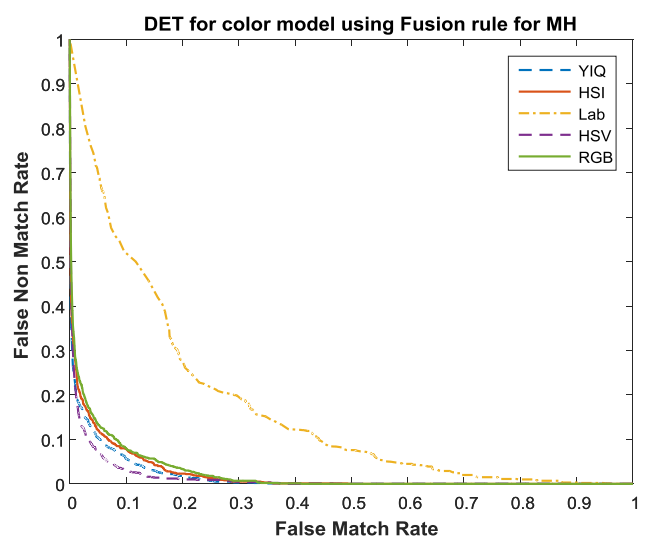

Fig 5. DET curves for various color models using MHD

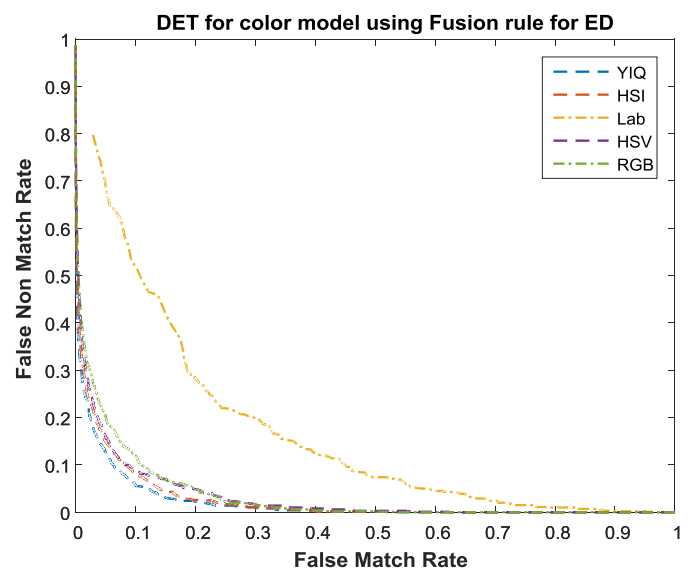

Fig 6. DET curves for various color models using ED
The Table 5 shows the comparison of the authentication accuracy of different methods presented by authors. The accuracy achieved by Hosseine et.al., [20] is $91.37 \%$. P. Radu et. al. [21] uses a fusion of red, green channel and the intensity channel of HSI model provides accuracy of $91.67 \%$. C. Sun et. al., [13] varies the value from 0 to 1 for red channel only and does fusion of all these values providing an accuracy of $90.7 \%$. In the proposed method fusion of score level using the sum rule is done for all channels of HSV model. The accuracy achieved by the proposed method is $92.1 \%$.

Table 5. Comparison of accuracy of UBIRIS database.

\begin{tabular}{|c|c|}
\hline Author & Accuracy \\
\hline C. Sun et. al.[13] & $\mathbf{9 0 . 7 0 \%}$ \\
\hline Hossein et. al.[20] & $\mathbf{9 1 . 3 7 \%}$ \\
\hline P. Radu et. al.[21] & $\mathbf{9 1 . 9 6 \%}$ \\
\hline Proposed method & $\mathbf{9 2 . 1 0 \%}$ \\
\hline
\end{tabular}

\section{CONCLUSIONS}

The paper introduced color histogram based approach for color iris authentication based on color iris feature extracted using different color models. The fusion of score level is implemented on color features extracted from color iris images acquired in noisy environment. The proposed method of color histograms deals with different image sizes, colors, eyelashes, eyelids and illuminations. The proposed algorithm yields better performance when compared with Sun [13], Hossein [20] and Radu [21]. The experimental results have demonstrated that the proposed method achieves better performance in accuracy and speed. Based on above experimentation HSV and YIQ color spaces are powerful as compared to RGB, YUV, HIS, HSL, Lab, Luv, LCH, and $\mathrm{YCbCr}$ color spaces for iris authentication. Experimental results demonstrate the effectiveness of HSV color space. The performance of the method using the HSV color model is very simple, effective, efficient and faster. The HSV color model is best model as compared to others which is suitable for color iris authentication. The proposed method achieves accuracy of $92.1 \%$, EER of 0.072 and computational time is $0.039 \mathrm{Sec}$. as compared to other methods. Experimental results suggest that the combination of HSV channels, its histogram and matching score fusion using weighted sum rule is more promising. The accuracy of the proposed system can further be improved by developing new models for feature extraction which are more efficient in terms of accuracy and EER.

\section{REFERENCES}

[1] A. Jain, R. Bolle, and S. Pankanti, Biometrics: Personal Identification in a Network Society. MA: Kluwer Academic Publisher, Norwell, 1999.

[2] J. Daugman, "High confidence recognition of persons by a test of statistical independence," IEEE Transactions on Pattern Analysis and Machine Intelligence, vol. 15, no. 11, pp. 1148-1161, 1993.

[3] J. G. Daugman, "How iris recognition works," IEEE Transactions on Circuits and Systems for Video Technology, vol. 14, no. 1, pp. 21-30, 2004.

[4] R. Wildes, "Iris recognition: an emerging biometric technology," in Proceedings of the IEEE Colloquium, vol. 85, no. 9, January 1997, pp. 1348-1363.

[5] W. W. Boles and B. Boashash, "A human identification technique using images of the iris and wavelet 
transform," IEEE Transactions on Signal Processing, vol. 46, no. 4, pp. 1185-1188, 1998.

[6] L. Ma, T. Tan, D. Zhang, and Y. Wang, "Local intensity variation analysis for iris recognition," IEEE Transactions on Pattern recognition, vol. 37, no. 6, pp. 1287-1298, 2003.

[7] X. Liu, K. W. Bowyer, and P. J. Flynn, "Experimental evaluation of iris recognition," in Proceedings of the 2005 IEEE computer society conference on computer vision and pattern recognition(CVPR05), June 2005, pp. 158-161.

[8] H. Proenca and L. A. Alexandre, "UBIRIS: A noisy iris image database," in Proceedings of the 13th International Conference on Image Analysis and Processing (ICIAP2005), September 2005, pp. 970-977, http://iris.di.ubi.pt.

[9] S. P. Narote, A. S. Narote, and L. M.Waghmare, "An automated iris image localization in eye images used for personal identification," in 14th International conference on advanced computing 'ADCOM 2006', Suratkal, November 2006, pp 250-253.

[10] E. Krichen, S. Garcia-Salicetti, and B. Dorizzi, "A new phase-correlation based iris matching for degraded images," IEEE Systems, Man, and Cybernetics, Part B: Cybernetics, vol. 39, no. 4, pp. 924-934, 2009.

[11] C. Boyce, A. Ross, M. Monaco, L. Hornak, and X. Li, "Multispectral iris analysis: A preliminary study," in Proceedings of the Conference on Computer Vision and Pattern Recognition Workshop, June 2006, pp. 51-55.

[12] M. K. Monaco, "Color space analysis for iris recognition," Master's thesis, Lane Department of Computer Science and Electrical Engineering, Morgan town, West Virginia, 2007.

[13] C. Sun, F. Melgani, C. Zhou, D. N. Francesco, L. Zhangand, and X. Liu, "Semi-supervised learning based color iris recognition," in Fourth International Conference on Natural Computation, vol. 4, 2008, pp. 242-249.

[14] F. S. Al-Qunaieer and L. Ghouti, "Color iris recognition using hypercomplex gabor wavelets," in 2009 Symposium on Bio-inspired Learning and Intelligent Systems for Security, August 2009, pp. 18-19.

[15] T. Tan, X. Zhang, Z. Sun, and H. Zhang, "Noisy iris image matching by using multiple cues," Pattern Recognition Letters, vol. 38, no. 5, pp. 5940- 5946, 2011.

[16] F. N. Sibai, H. I. Hosani, R. M. Naqbi, and S. D. S. Shehhi, "Iris recognition using artificial neural networks," Expert Systems with Applications, vol. 38, no. 5, pp. 5940-5946, 2011.

[17] E. L. Broek, "Human-centered content-based image retrieval," Ph.D. dissertation, Nijmegen Institute for Cognition and Information (NICI), Radboud University Nijmegen, Netherlands, 2005.

[18] J. Kittler, M. Hatef, R. P. Duin, and J. G. Matas, "On combining classifiers," IEEE Transactions on Pattern Analysis and Machine Intelligence, vol. 20, no. 3, pp. 226-239, 1998.

[19] A. Ross and A. K. Jain, "Information fusion in biometrics," Pattern Recognition Letter, vol. 24, no. 13, pp. 2115-2125, 2003.

[20] M. S. Hosseini, B. Araabi, and H. Soltanian-Zadeh, "Pigment melanin: Pattern for iris recognition," IEEE Transactions on Instrumentation and Measurement, vol. 59, no. 4, pp. 792-804, 2010.

[21] P. Radu, K. Sirlantzis, W. Howells, F. Deravi, and S. Hoque, "Information fusion for unconstrained iris recognition," International Journal of Hybrid Information Technology, vol. 4, no. 4, pp. 1-11, 2011. 